\title{
Design and implementation of Wavelet Transform using Adaptive Genetic Algorithms and Analog filters*
}

\author{
$\mathrm{Mu} \mathrm{Li}^{1}$, Yigang $\mathrm{He}^{2}$, Yangliang $\mathrm{Ou}^{1}$, Junnian Wang ${ }^{1}$ \\ ${ }^{1}$ College of Information and Electrical Engineering, Hunan University of Science and Technology, Hunan, China \\ ${ }^{2}$ College of Electrical and Information Engineering, Hunan University, Hunan, China \\ limuucn@163.com,yghe@hnu.cn
}

\begin{abstract}
This paper presents a low power and low-voltage analog switched-current (SI) filters implementation of wavelet transform (WT) for real-time requirements in signal processing. First, an adaptive genetic algorithm (AGA) is used to calculate the transfer function of the filters, whose impulse response is the required wavelet base. This approach improves the approximation performance than the previous traditional approaches and allows for the circuit implementation of any other wavelet base. Next, the approximation wavelet function is implemented using SI filters based on the cascade structure with SI differentiators as main building blocks. The Gaussian wavelet is selected as an example to illustrate the design procedure. Simulations demonstrate that the proposed method implements WT is an excellent way.

Index Terms - Wavelet transform, analog filters, analog implementation, switched-current differentiators, adaptive genetic algorithms.
\end{abstract}

\section{I . Introduction}

The WT has been a very promising mathematical tool for signal processing, due to its good estimation of time and frequency localizations [1]. The WT is traditionally implemented using digital circuits. However, for low-power, low-voltage and real-time applications, it is not suitable to implement the WT due to the high power consumption and large area associated with the required A/D (analog-digital) converter. Consequently, the analog WT implementations have been an attractive field in signal processing.

Recently, some significant advances for implementing WT with analog filters have been introduced in the literatures [211]. Among, the implementations using analogue sampled-data circuits have attracted much attention [2, 8-11]. A key feature of using these circuits for implementing WT is that dilation constant across different scales may be easily and precisely controlled by the clock frequencies. Typically, the switchedcapacitors (SC) circuit has been used to implement analog WT [2]. However, the SC circuits are not fully compatible with current trends in digital CMOS process and their performance suffers as supply voltages are scaled down. To resolve these problems, the SI circuit [8-11] is applied to implement WT. The SI WT circuits consist of analog filters whose impulse response is the approximated wavelet and its dilations. So the performance of the WT realization depends largely on the approximation accuracy of the wavelet function. In the proposed approximation methods, Padé approximation [3, 8] is employed to approximate the Laplace transform of the desired wavelet filter transfer function. However, (1) the stable transfer function of a wavelet filter does not automatically result from the Padé approximation, (2) the choice of the degrees for the numerator and denominator polynomials in the approximation transfer function is difficult, (3) the wavelet approximation is only obtained directly in the Laplace domain, but not in the time domain. $L_{2}$ approximation $[4,5,6,9]$, in order to find a suitable wavelet base approximation and improves the approximation accuracy compared to Padé is proposed. However, because of the existence of local optima in this approximation, the performance greatly depends on the selection of the approximation starting point. Furthermore, in the WT circuit, as compared to SI integrators [8-11], litter attention has been paid to applying SI differentiators in analog WT systems. However, SI differentiators have many advantages such as good noise rejection and stability.

In this paper, a new method of implementing WT using AGA and SI filters is presented. The universal approximation function of the wavelet base is constructed by a parameterized class of the filter impulse response function. The optimum parameters of the wavelet approximation function are obtained using AGA. The WT circuits are composed of analog SI filter banks whose impulse response is the wavelet approximation function and its dilations. The filter design employs the cascade structure with SI differentiators as main building blocks. The simulation results demonstrate the proposed method is effective for implement WT in analog way.

\section{II . Analog wavelet transform implementation}

The WT is a linear operation that decomposes a signal into components that appear at different scales [1]. The WT of a function at scale $a$ and position $\tau$ is given by

$$
W T_{f}(a, \tau)=\frac{1}{\sqrt{a}} \int f(t) \phi\left(\frac{t-\tau}{a}\right) d t=f(t) * \frac{1}{\sqrt{a}} \phi\left(\frac{-t}{a}\right)
$$

where $\phi(t)$ is the wavelet base and $*$ denotes convolution. It is well known that when a signal $f(t)$ is passed through a linear filter, the filter output is the convolution of $f(t)$ with the impulse response $h(t)$ of the filter. Therefore, we can see that

\footnotetext{
* This work was supported by the National Natural Science Funds of China for Distinguished Young Scholar Grant \#50925727, the Scientific Fund of Hunan Provincial Education Department Grant \#10C0672, the Xiangtan Science and Technology Foundation of China Grant \#CG20121006, the Students Research Innovative of Hunan University of Science and Technology Grant \#SYZ2012038, and the Open Fund Project of Hunan Province University Innovation Platform \#11K027.
} 
the analog computation of $W T_{f}(a, \tau)$ can be achieved through the implementation of an analog filter of which the impulse response satisfies $h(t)=(1 / \sqrt{a}) \phi(-t / a)$. The first derivative of a Gaussian WT system has been selected to illustrate the design procedure in this paper.

\section{III . Wavelet function approximation}

\section{A. Approximation model Design}

A given wavelet function will usually be non-causal, in order to implement the wavelet filter, one must first use a suitable approximation method. In the work, the approximation of the first derivative of a Gaussian wavelet in time domain is presented. The first derivative of a Gaussian wavelet is given by

$$
\phi(t)=-1.7865 t e^{-t^{2}}
$$

This wavelet function is non-causal and it can not be directly implemented using the hardware circuit. Note that $h(t)$ will then be zero for negative $t$, so that the wavelet function $\phi(t)$ which does not have this property must be time-shifted to facilitate an accurate approximation of $W T_{f}(a, \tau)$. If it chooses the time-shift $t_{0}=2$, this gives rise to the following time-reversed and time-shifted wavelet function

$$
\phi\left(t_{0}-t\right)=\phi(2-t)=-1.7865(2-t) e^{-(2-t)^{2}}
$$

As stated before, if $h(t)$ is used to approximate a time-shifted and time-reversed wavelet function $\phi(2-t)$, the output of the filter is the approximate wavelet transform $W T_{f}(a, \tau)$ of the input signal. The quality of such implementations depends on the accuracy of the corresponding wavelet approximations. For the generic situation of stable systems with distinct poles, the impulse response $h(t)$ may typically have the universal form. For instance, if a 8th order approximation is considered, this function $h(t)$ may be expressed by

$$
\begin{aligned}
h(t)= & \lambda_{1} e^{\lambda_{2} t} \sin \left(\lambda_{3} t\right)+\lambda_{4} e^{\lambda_{2} t} \cos \left(\lambda_{3} t\right)+\lambda_{5} e^{\lambda_{6} t} \sin \left(\lambda_{7} t\right)+ \\
& \lambda_{8} e^{\lambda_{6} t} \cos \left(\lambda_{7} t\right)+\lambda_{9} e^{\lambda_{10} t} \sin \left(\lambda_{11} t\right)+\lambda_{12} e^{\lambda_{10} t} \cos \left(\lambda_{11} t\right)+ \\
& \lambda_{13} e^{\lambda_{14} t} \sin \left(\lambda_{15} t\right)+\lambda_{16} e^{\lambda_{14} t} \cos \left(\lambda_{15} t\right)
\end{aligned}
$$

where $\lambda_{i},(i=1,2, \cdots, 15)$ is the real coefficient. Among, the parameters $\lambda_{2}, \lambda_{6}, \lambda_{10}$ and $\lambda_{14}$ must be strictly negative for reasons of stability. The error integral is defined as

$$
\|h(t)-\phi(2-t)\|^{2}=\int_{0}^{\infty}[h(t)-\phi(2-t)]^{2} d t
$$

The sums of squares error of the discrete points are expressed by

$$
E(\lambda)=\sum_{m=1}^{700}[h(m \Delta t)-\phi(2-m \Delta t)]^{2}
$$

where $\Delta t$ is the sampling time interval and $m$ is the sampling points. In order to calculate the optimal parameters of the approximation $h(t)$, the optimal model for approximating $h(t)$ is described as

$$
\left\{\begin{array}{l}
\min \left(E(\lambda)=\sum_{m=1}^{700}[h(m \Delta T)-\phi(2-m \Delta T)]^{2}\right) \\
\text { s.t. } \quad \lambda_{k}<0,(k=2,6,10,14)
\end{array}\right.
$$

From equation (7), it is easy to know that this is a typical nonlinear optimization problem with nonlinear constants. Hence, the global intelligent optimization algorithms are described and employed to calculate the optimal values of the model in the next section.

\section{B. Parameters optimization using AGA}

Genetic algorithms (GA) have been widely used in various fields such as pattern recognition, combination optimization, and machine learning due to their global optimization property. Especially in dealing with nonlinear problems, GA has the superior performances than other traditional optimization methods [12]. Assume that the population is $P(g)$ of feasible solution space, where $g$ is the evolution generation. Population size is $N$, so $g$ generation population can be expressed as $X^{g}=\left[x_{1}^{g}, x_{2}^{g}, \cdots, x_{N}^{g}\right]^{T}$, whose $i$ th individual can be described as $x_{i}^{g}=\left[\lambda_{1 i}^{g}, \lambda_{2 i}^{g}, \lambda_{3 i}^{g}, \cdots, \lambda_{16 i}^{g}\right]^{T}$ associated with the coefficients of the approximation model. The basic framework of AGA is presented to calculate the coefficients as follows:

1) Initialize a population of $N$ individuals with random values generated according to a uniform probability distribution in the $D$ dimensional problem space. Specify the population size $N=10$, the sampling time interval $\Delta t=0.01$. Set the maximum evolution generation $G_{\max }=9000$ and the initial generation $g=0$.

2) Calculate the fitness value of the chromosome according to the given objective function. The greater the fitness of a chromosome can be survived. The objective function is given by

$$
F(\lambda)=\sum_{m=1}^{700}[h(0.01 m)-\phi(2-0.01 m)]^{2}
$$

3) Given the crossover probability, the crossover agnate generates the new offspring. To ensure that the algorithm can obtain the optimal solution, we give a reconfigurable strategy for the probability of the crossover. The probabilities of the crossover is described as [12]

$$
P_{c}=\left\{\begin{array}{l}
C_{1}+\left(C_{2}-C_{1}\right) *\left(f_{\max }-f_{c}\right) /\left(f_{\max }-f\right), f_{c} \geq f_{\text {avg }} \\
C_{2}, f_{c}<f_{\text {avg }}
\end{array}\right.
$$

where $C_{1}, C_{2} \in(0,1) . f_{c}$ is the bigger fitness in two individuals of the crossover. $f_{\max }$ is the maximum fitness of the current population. $f_{\text {avg }}$ is the average fitness of the current population.

4) Given the mutation probability, the mutation occurs in a certain place of a chromosome and a new individual is born. The probabilities of the mutation is expressed as [12]

$$
P_{m}=\left\{\begin{array}{l}
M_{3}+\left(M_{4}-M_{3}\right) *\left(f_{\max }-f_{i}\right) /\left(f_{\max }-f_{\text {avg }}\right), f_{i} \geq f_{\text {avg }} \\
M_{4}, f_{i}<f_{\text {avg }}
\end{array}\right.
$$

where $M_{3}, M_{4} \in(0,1) . f_{i}$ is the fitness of the $i$ th individual in the population. 
5) Choose pairs of individuals from the population in such a way that those with higher fitness will get more copies. In this paper, the roulette wheel selection is applied.

6) If the termination condition is satisfied, the algorithm ends, otherwise return to Step 2.

According to the above steps of AGA, the problem in equation (7) can be solved. The accurate globe optimal solutions $\lambda_{i}$ of $h(t)$ are given in Table I. After transforming the time domain approximation function, the 8th Laplace transfer function of the first derivative of a Gaussian wavelet filter (scale $a=1$ ) is obtained as

TABLE I The Optimal Solutions of the Gaussian Wavelet Approximation

\begin{tabular}{|c|c|c|c|c|c|c|c|}
\hline $\mathrm{i}$ & $\lambda_{\mathrm{i}}$ & $\mathrm{i}$ & $\lambda_{\mathrm{i}}$ & $\mathrm{i}$ & $\lambda_{\mathrm{i}}$ & $\mathrm{i}$ & $\lambda_{\mathrm{i}}$ \\
\hline 1 & -42.215 & 5 & 4.4321 & 9 & 0.5156 & 13 & 11.8121 \\
\hline 2 & -1.5281 & 6 & -1.1450 & 10 & -0.9640 & 14 & -1.2386 \\
\hline 3 & -0.1712 & 7 & 2.6092 & 11 & -3.9569 & 15 & -1.4180 \\
\hline 4 & 1.1941 & 8 & -0.5131 & 12 & 0.1789 & 16 & -0.9251 \\
\hline
\end{tabular}

The other transfer function $H_{a}(s)$ of the wavelet filters at scales $a$ can be derived from equation (11) by the theory of Laplace transfer. Fig.1 shows the 8th approximation performance of the first derivative of a Gaussian wavelet using different methods. Obviously, the performance of AGA is better than $L_{2}$ and Padé methods.

$$
\begin{aligned}
H_{1}(s)= & \frac{-0.0653 s+7.9013 e-4}{s^{2}+2.2902 s+8.1157} \cdot \frac{s^{2}+12.7941 s+34.7455}{s^{2}+1.9278 s+16.5883} . \\
& \frac{s^{2}+2.7941 s+39.7447}{s^{2}+2.4779 s+3.5474} \cdot \frac{s^{2}-1.7951 s+19.5036}{s^{2}+3.0555 s+2.3637}
\end{aligned}
$$

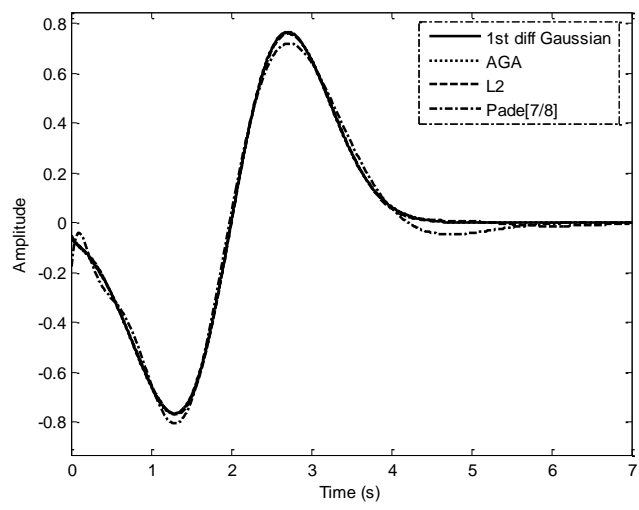

Fig. 1 The approximation of the Gaussian wavelet.

\section{Wavelet Filter Design using SI Circuit}

The SI technique presents, as the main advantages over SC filters, simple implementation on a digital CMOS process, less chip area and power, insensitivity to nonlinearities in capacitances and transconductances, and circuits that can operate at higher frequency [13]. According to the filters design theory, higher order filters can easily be realized using the biquads. In this paper, the SI WT filter design is based on the cascade architecture with SI differentiator as the main building blocks.

The general SI biquad differentiator [14] is used, whose transfer function in $z$-domain is given by

$$
\begin{aligned}
& H(z)=I_{0}(z) / I_{i}(z) \\
& =\frac{-\left[\left(\alpha_{2} \alpha_{3}+\alpha_{1} \alpha_{3}\right) z^{2}+\left(\alpha_{0}-\alpha_{1} \alpha_{3}-2 \alpha_{2} \alpha_{3}\right) z+\alpha_{2} \alpha_{3}\right]}{\alpha_{3} \alpha_{5} z^{2}+\left(1+\alpha_{4}-2 \alpha_{3} \alpha_{5}\right) z+\left(\alpha_{3} \alpha_{5}-\alpha_{4}\right)}
\end{aligned}
$$

Consider the continuous-time biquad described by

$$
H(s)=-\frac{k_{2} s^{2}+k_{1} s+k_{0}}{s^{2}+\left(\omega_{0} / Q\right) s+\omega_{0}^{2}}
$$

Applying the bilinear $z$-transform gives

$$
\begin{aligned}
& H(z)=-\left\{\left[\left(4 k_{2}+2 k_{1} T+k_{0} T^{2}\right) / D\right] z^{2}+\right. \\
& \left.\left[\left(2 k_{0} T^{2}-8 k_{2}\right) / D\right] z+\left[\left(4 k_{2}-2 k_{1} T+k_{0} T^{2}\right) / D\right]\right\} / \\
& \left\{\left[\left(\omega_{0} T^{2}+2 \omega_{0} T / Q+4\right) / D\right] z^{2}+\left[\left(2 \omega_{0}^{2} T^{2}-8\right) / T\right] z+1\right\}
\end{aligned}
$$

Comparing coefficients of equation (12) and (14) gives

$$
\begin{aligned}
& \alpha_{0}=k_{0} / \omega_{0}^{2} ; \alpha_{1} \alpha_{3}=k_{1} / T \omega_{0}^{2} ; \\
& \alpha_{2} \alpha_{3}=\left(k_{0}-2 k_{1} / T+4 k_{2} / T^{2}\right) / 4 \omega_{0}^{2} ; \\
& \alpha_{4}=1 / Q T \omega_{0} ; \alpha_{5}=\left(4 / T^{2}+2 \omega_{0} / Q T+\omega_{0}^{2}\right) / 4 \omega_{0}^{2}
\end{aligned}
$$

Equation (11) are used to calculate the transistor aspect ratios $(W / L)\left(\alpha_{0}-\alpha_{5}\right)$ to be used in the SI differentiators, giving the required the transfer function $H_{1}(s)$. For this example, the transistor aspect ratios of the SI differentiator realization are given in Table II. Four biquad sections in cascade structure are to be connected to realize the approximation transfer functions. The circuit of eighth order wavelet filters using SI differentiators in ASIZ software is given in Fig. 2.

TABLE II The Optimal Solutions of the Gaussian Wavelet Approximation

\begin{tabular}{|c|c|c|c|c|}
\hline$\alpha_{i}$ & $\begin{array}{c}\text { First } \\
\text { section }\end{array}$ & $\begin{array}{c}\text { Second } \\
\text { section }\end{array}$ & $\begin{array}{c}\text { Third } \\
\text { section }\end{array}$ & $\begin{array}{c}\text { Fourth } \\
\text { section }\end{array}$ \\
\hline$\alpha_{0}$ & $9.736 \mathrm{e}-5$ & 2.09458 & 11.20390 & 8.25130 \\
\hline$\alpha_{1}$ & 0.03218 & 3.08509 & 3.15059 & 3.03778 \\
\hline$\alpha_{2}$ & 0.01611 & 0.05437 & 5.73602 & 10.35077 \\
\hline$\alpha_{3}$ & 1.00000 & 1.00000 & 1.00000 & 1.00000 \\
\hline$\alpha_{4}$ & 1.12878 & 0.46486 & 2.79405 & 5.17070 \\
\hline$\alpha_{5}$ & 2.78587 & 1.44697 & 6.15737 & 9.60440 \\
\hline
\end{tabular}
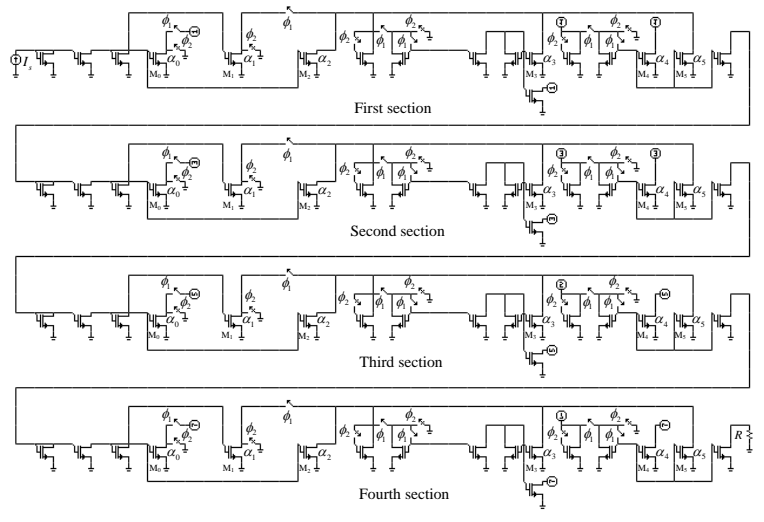

Fig. 2 The Gaussian WT circuit with SI differentiator 


\section{Simulation Results}

The whole circuit has been simulated using the ASIZ simulator [14]. Since the dilations of a given SI filter may be controlled by changing the clock frequency, so adjusting the various clock frequencies of the circuits with the same system architecture, one can obtain different scale wavelet functions. Defining $I_{s}=1 \mathrm{~A}, R=1 \Omega$ and clock frequency is $100 \mathrm{kHz}, 50 \mathrm{kHz}$, $25 \mathrm{kHz}$ and $12.5 \mathrm{kHz}$, respectively. Fig. 3 shows the impulse response of the wavelet filters with four scales. The simulated impulse response waveforms of the different scale filters achieve the negative peak value $0.1769 \mathrm{~A}$ at $0.04 \mathrm{~ms}, 0.08 \mathrm{~ms}$, $0.16 \mathrm{~ms}$ and $0.32 \mathrm{~ms}$, respectively, which are different from the normalized values of the original waveform. The approximation quality of the first derivative of a Gaussian wavelet is excellent to the ideal wavelet function, which confirms the performance of the SI wavelet filters. Only changing $W / L$ of the output current mirror, the output gain of the waveform will be adjusted for the practice applications.

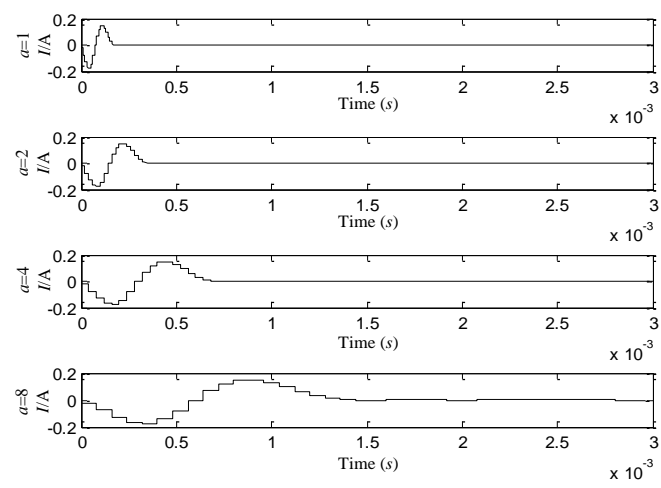

Fig. 3 Simulated impulse responses of the Gaussian filter with four scales.

In order to analyse the effect of finite $G_{m} / G_{d s}$ ratios in the transistors and parasitic $C_{g d}$ capacitances, another simulation is made. Assuming $G_{m} / G_{d s}$ and $C_{g s} / C_{g d}$ ratios of 2000, with the biasing current sources assumed as ideal, the frequency response of the wavelet filter is shown in Fig. 4. The simulated frequency response is closed to the ideal frequency response, which indicates that the designed system has litter effect in the sensitivity to the imperfections.

\section{Conclusions}

A low voltage and low power analog circuit for implementing the WT using AGA and SI filters is presented. The wavelet base is approximated by AGA method in the time domain. This approximation approach performs well in the practice that it leads to relatively low order approximation. Then the approximating wavelet function is implemented in analog SI filter, based on SI differentiator as main building block. By changing the clock frequency, the SI filter obtains the various scales wavelet function to implement WT. Simulation results the proposed method is effective. Furthermore, from the results obtained, we may deduce this procedure could very well be used to implement other wavelet bases.

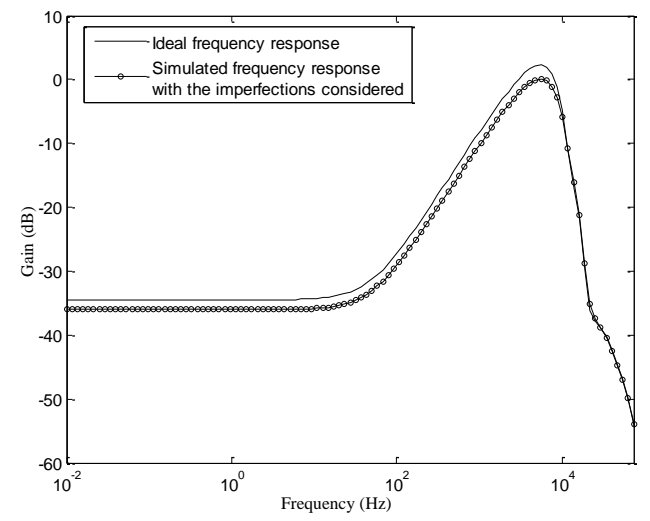

Fig. 4 Simulated frequency response of the Gaussian filter with the imperfections considered $(a=1)$.

\section{References}

[1] R. X. Gao, R. Q. Yan, Wavelets: Theory and Applications for Manufacturing, New York: Springer-Verlag Press, 2010.

[2] J. Lin, W. H. Ki, T. Edwards, S. Shamma, "Analog VLSI implementations of auditory wavelet transforms using switchedcapacitor circuits," IEEE Transactions on Circuits and Systems, vol. 41, no. 9, pp. 572-583, 1994.

[3] S. A. P. Haddad, B. Sumit, W. A Serdijn, "Log-domain wavelet bases," IEEE Transactions on Circuits and Systems, vol. 52, no. 10, pp. 20232032, 2005.

[4] P. R. Agostinho, S. A. P. Haddad, , J. A. DE Lima, W. A. Serdijn, "An ultra low power CMOS PA/V transconductor and its application to wavelet filters," Analog Integrated Circuits and Signal Processing, vol. 57, no. 1-2, pp. 1-14, 2008.

[5] J. M. H. Karel, S. A. P. Haddad, S. Hiseni, R. L. Westra, W. A. Serdijn, R. L. M. Peeters, "Implementing wavelets in continuous-time analog circuits with dynamic range optimization," IEEE Transactions on Circuits and Systems, vol. 59, no. 1, pp. 2023-2032, 2012.

[6] A. N. Akansu, W. A. Serdijn, W. Selesnick, "Emerging applications of wavelets: A review," Physical Communication, vol. 3, no. 1, pp. 1-18, 2010.

[7] W. Zhao, Y. Sun, Y. HE, "Minimum component high frequency Gm-C wavelet filters based on Maclaurin series and multiple loop feedback," Electronic Letters, 2010, vol. 46, no. 1, pp. 34-36.

[8] Q. C. Hu, Y. G. He, M. R. Liu. "Design and implementation of wavelet filter using switched-current circuits," In Proceedings of the IEEE TENCON, Melbourne, Qld, pp. 1-6, 2005.

[9] W. S. Zhao, Y. G. He. "Realization of wavelet transform using switched-current filters," Analog Integrated Circuits and Signal Processing, vol. 71, no. 3, pp.571-581, 2012.

[10] M. Li, Y. G. He, "Analogue implementation of wavelet transform using discrete time switched-current filters," In Proceedings of the International Conference on Electric and Electronics, Nanchang, Jiangxi, pp. 677-682, 2011.

[11] M. Li, Y. G. He, "Analog VLSI implementation of wavelet transform using switched-current circuits," Analog Integrated Circuits and Signal Processing, vol. 71, no. 2, pp. 283-291, 2012.

[12] J. F. Yu, Y. H. Yin, "Assembly line balancing based on an adaptive genetic algorithm," International Journal of Advanced Manufacturing Technology, vol. 48, no. 1-4, pp. 347-354, 2010.

[13] J. B. Hughes, I. C. Macbeth, D. M. Pattullo, "Switched current filters," IEE Proceedings G, Circuits, Devices and Systems, vol. 137, no. 2, pp. 156-162, 1990.

[14] C. Toumazou, J. B. Hughes, and N. C. Battersby, Switched-Currents: an Analogue Technique for Digital Technology. Exeter: Short Run Press, 1993. 